\title{
Runaway Electron experiments at COMPASS in support of the EUROfusion ITER physics research
}

\author{
J Mlynar ${ }^{1,2}$, O Ficker ${ }^{1,2}$, E Macusova ${ }^{1}$, T Markovic ${ }^{1,3}$, D \\ Naydenkova $^{1}$, G Papp ${ }^{4}$, J Urban ${ }^{1}$, M Vlainic ${ }^{5,6}, \mathbf{P}$ Vondracek $^{1,3}$, \\ V Weinzettl ${ }^{1}$, O Bogar ${ }^{1,7}$, D Bren ${ }^{2}$, D Carnevale ${ }^{8}$, A Casolari ${ }^{1}$, \\ J Cerovsky ${ }^{1,2}, \mathbf{M}$ Farnik $^{1,2}, \mathbf{M}$ Gobbin $^{8}, \mathbf{M}$ Gospodarczyk $^{9}, \mathbf{M}$ \\ Hron $^{1}$, P Kulhanek ${ }^{1,10}$, J Havlicek ${ }^{1}$, A Havranek ${ }^{1,10}$, M \\ Imrisek $^{1,3}, \mathbf{M}$ Jakubowski $^{11}$, $\mathbf{N}$ Lamas $^{12}, \mathrm{~V}$ Linhart $^{2}, \mathbf{K}$ \\ Malinowski ${ }^{11}$, M Marcisovsky ${ }^{2}$, E Matveeva ${ }^{1}$, R Panek ${ }^{1}$, V V \\ Plyusnin $^{13}$, M Rabinski ${ }^{11}$, V Svoboda ${ }^{2}$, P Svihra ${ }^{2}$, J Varju ${ }^{1}$, J \\ Zebrowski ${ }^{11}$, the COMPASS Team and the EUROfusion MST1 \\ Team $^{\dagger}$ \\ ${ }^{1}$ Institute of Plasma Physics of the CAS, CZ-18200 Praha 8, Czech Republic \\ ${ }^{2}$ FNSPE, Czech Technical University in Prague, CZ-11519 Praha 1, Czech Republic \\ ${ }^{3}$ FMP, Charles University, Ke Karlovu 3, CZ-12116 Praha 2, Czech Republic \\ ${ }^{4}$ Max Planck Institute for Plasma Physics, Garching D-85748, Germany \\ ${ }^{5}$ Department of Applied Physics, Ghent University, Ghent 9000, Belgium \\ ${ }^{6}$ Institute of Physics, University of Belgrade, P.O. Box 68, 11080 Belgrade, Serbia \\ ${ }^{7}$ Faculty of Mathematics, Physics and Informatics, Comenius University in \\ Bratislava, 84248 Bratislava, Slovakia \\ ${ }^{8}$ Consorzio RFX, Corso Stati Uniti 4, 35127 Padova, Italy \\ ${ }^{9}$ Universita di Roma Tor Vergata, 00133 Roma, Italy \\ ${ }^{10}$ FEE, Czech Technical University in Prague, CZ-12000 Praha 2, Czech Republic \\ ${ }^{11}$ National Centre for Nuclear Research (NCBJ), Otwock-Swierk, Poland \\ ${ }^{12}$ University of Lorraine, 54000 Nancy, France \\ ${ }^{13}$ Centro de Fusao Nuclear, IST, Lisbon, Portugal \\ † See the author list of H. Meyer et al., Nuclear Fusion 57 (2017) 102014 \\ E-mail: mlynar@ipp.cas.cz
}

\begin{abstract}
.
The role of the COMPASS tokamak in research of generation, confinement and losses of Runaway Electron (RE) population is presented. Recently, two major groups of experiments aimed at improved understanding and control of the REs have been pursued. First, the effects of the Massive Gas Injection (MGI, $\sim 10^{21} \mathrm{Ar} / \mathrm{Ne}$ particles) and impurity seeding ( $\sim 10^{18}$ particles) were studied systematically. The observed phenomena include generation of the post-disruption RE beam and current conversion from plasma to RE. Zero loop voltage control was implemented in order to study the decay in simplified conditions. A distinctive drop of background plasma temperature and electron density was observed following an additional deuterium injection into the RE beam. The loop voltage control the parametric dependence of the current decay rate $d I / d t$ can be studied systematically and possibly extrapolated to larger
\end{abstract}


facilities. Second, recent results of experiments focused on the role of the magnetic field in physics of $\mathrm{RE}$ were analysed. In this contribution, special attention is given to the observed effects of the Resonant Magnetic Perturbation (RMP) on the RE population. The benefits of the RE experiments on COMPASS was reinforced by diagnostic enhancements (fast cameras, Cherenkov detector, vertical ECE etc.) and modelling efforts (in particular, coupling of the METIS and LUKE codes).

Keywords: tokamaks, runaway electrons, MHD instabilities, disruptions

\section{Introduction}

Thermal equilibrium in plasmas can be disturbed, among others, by applying external electric field that accelerates in particular the light electrons. Up to a certain electric field intensity, the acceleration is mitigated by collisional drag. However, there exists a critical electric field [1] above which net acceleration of the high energy part of the electron thermal distribution is possible, which generates a highly directional population of non-thermal electrons denoted by the term Runaway Electrons (RE). The process described above is the most common mechanism of the primary RE generation, usually referred to as Dreicer generation [2]. In the classical model with full relativistic treatment [1] the critical electric field is

$$
E_{\mathrm{c}}=\frac{e^{3} n_{\mathrm{e}} \ln \Lambda}{4 \pi \epsilon_{0}^{2} m_{\mathrm{e}} c^{2}} .
$$

The critical field increases with plasma density $n_{e}$, and is only a weak function of temperature through the Coulomb logarithm $\ln \Lambda$. A more detailed introduction into the primary RE generation can be found e.g. in the paper by Granetz et al. [3]. Relation of the critical field to the experiments will be mentioned in section 2 .

$\mathrm{RE}$ are crucial in producing lightning discharges in thunderstorms [4]. In these events, the secondary mechanism of the RE production - the RE avalanche which may severely amplify the primary generation - plays a significant role. In the avalanche, close collisions between RE and thermal electrons (knock-on collisions) cause the latter to achieve sufficient velocity to become a part of the RE population, while the former often remain in the RE population [5], albeit with a lower velocity and/or a slightly modified trajectory [6]. In tokamaks, the RE generation is driven by the toroidal electric field induced in order to ramp-up and maintain toroidal electric current in plasma. The RE population may appear in practice in three different phases of the tokamak discharge, depending on its parameters:

(i) In the initial phase of the discharge, i.e. in the plasma breakdown, burn-through and the current ramp-up [7]. Primary energetic electrons (the RE seed) are generated within the initial ionisation and/or the burn-through phase due to the high level of the induced toroidal electric field, which remains elevated during the subsequent current ramp-up phase. While the field is above-critical (which can be controlled via fuelling) both the RE current and energy steadily increase. 
(ii) In the current plateau phase of the discharge, provided that the plasma density is sufficiently low [3, 8, 9] so that the toroidal electric field is above the critical field given by eq. (1). The case of these quiescent $R E$ discharges at COMPASS tokamak are mentioned in section 2 .

(iii) Following a disruption of the plasma discharge. The rapid thermal quench after the disruption results in a temporary accumulation of supra-thermal electrons due to their lower collisionality. The subsequent current quench increases the toroidal electric field that accelerates the supra-thermal electrons. This form of the primary $\mathrm{RE}$ generation is referred to as the hot-tail mechanism [10]. Modeling suggests that the above mentioned Dreicer generation mechanism can also convert a significant fraction of the predisruption plasma current into a RE seed [11, 12]. The RE seed generated by these primary generation mechanisms is further multiplied by avalanche [5], in particular in large devices [13]. Compton scattering of Hard Xrays on electrons and tritium decay would also contribute to the $\mathrm{RE}$ generation in large facilities [14].

In the current ramp-up phase, the RE population can be well controlled by the fuelling scenario [15] due to its low energy. The plateau phase is safe against RE generation for fusion relevant plasma densities. However, REs generated following a plasma disruption raise a serious issue for operation of the future tokamak fusion reactors [12, 16]. The highly undesirable, violent event of plasma disruption can incidentally occur in tokamak operation due to multiple reasons and produce major mechanical and heat shocks [17, 18]. The RE beam generated in a disruption can take over a substantial part of the original plasma current. At ITER it is predicted that the post-disruption RE beam with several tens of $\mathrm{MeV}$ can carry up to $70 \%$ of pre-disruptive plasma current [18. The RE beam could cause substantial damage to the first wall of ITER (for the damage in COMPASS see Fig. 1) and in an extreme case result in an in-vessel coolant leak. Therefore, unmitigated post-disruption RE beams are not acceptable in fusion reactors, including ITER.

The RE beams in tokamaks prove difficult to control [19] and challenging to mitigate by any external intervention as yet, which strongly stimulates research in the field of RE generation, confinement and losses. Unfortunately, the results are far from conclusive due to

- limited scope of the RE-relevant diagnostics [20, 21, 22, 23],

- highly demanding preconditions for theory and modelling, see e.g. [24, 25], and

- limited expertise in the position control of the RE beams [19].

In order to progress efficiently, the RE research efforts in Europe have been coordinated by the EUROfusion consortium within its ITER Physics Research programme which intermediates, among others, RE relevant experiments at tokamaks JET [26], AUG [27, 28], TCV [27, 29, 30], FTU [31] and COMPASS [32, 33, 34].

The COMPASS tokamak has been operating in Prague since 2008, with systematic contributions to $\mathrm{RE}$ research in dedicated experimental campaigns since 2014. The 


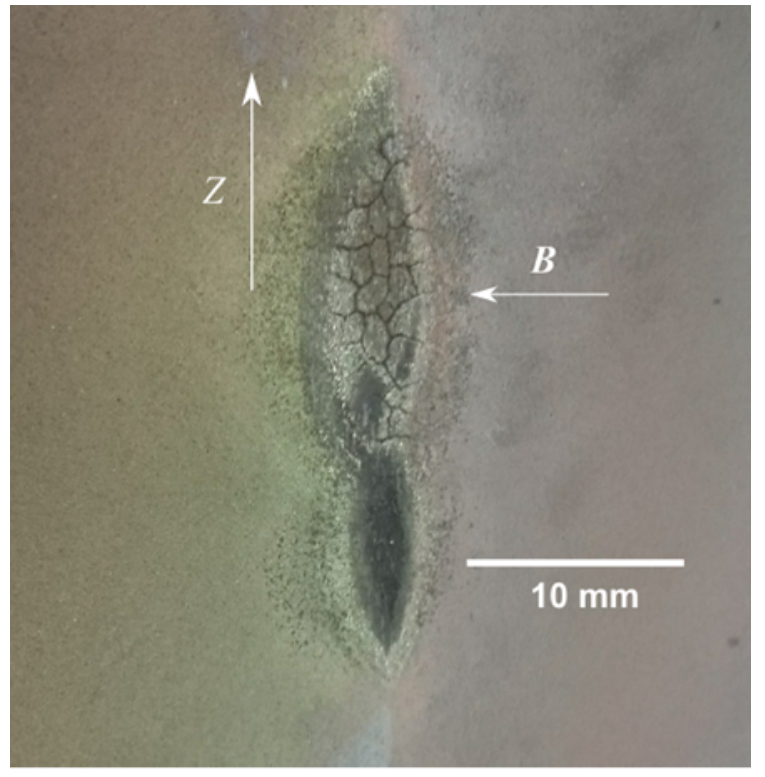

Figure 1. Wall damage due to interaction with Runaway Electrons (RE) at COMPASS. The damage was provoked on a protruding graphite inner limiter tile in several tens of RE discharges. The two locations of the damage correspond to two separate vertical positions of the RE beam in this set of experiments.

facility features ITER-like plasma shapes with major radius $R_{0}=0.56 \mathrm{~m}$ and minor radius $a=0.23 \mathrm{~m}$, typical toroidal field $B_{T}=0.9-1.5 \mathrm{~T}$, plasma current in the flat-top phase $I_{p}<350 \mathrm{kA}$, up to $600 \mathrm{~kW}$ neutral beam heating and a routine H-mode operation [35. Directions of the $B_{T}$ and $I_{p}$ can be changed flexibly and independently. Within work published in this contribution, the $B_{T}$ field as well as $I_{p}$ were oriented clockwise from the top view. The toroidal magnetic field was $B_{T}=1.15 \mathrm{~T}$ if not stated otherwise.

The following diagnostic tools have been instrumental in the RE experiments:

- rich magnetic diagnostics [36] consisting, among others, of three poloidal arrays of 24 internal pick-up (Mirnov) coils covering radial, poloidal and toroidal components, 16 internal partial Rogowski coils and 8 flux loops,

- fast visible cameras (Photron Mini UX100 and Photron SA-X2) and fast IR camera Telops FAST-IR 2K,

- a pair of unshielded $\mathrm{NaI}(\mathrm{Tl})$ scintillation detectors to observe hard X-ray (HXR) radiation (energy range approx. $50 \mathrm{keV}-10 \mathrm{MeV}$ ) and a composite $\mathrm{ZnS}(\mathrm{Ag}$ ) scintillation detector shielded by $10 \mathrm{~cm}$ of lead, therefore sensitive to neutrons and HXR approx. above $500 \mathrm{keV}$,

- $2 \mathrm{~mm}$ microwave interferometer with central vertical line of sight for monitoring and control of the plasma density,

- Thomson scattering (TS) data to determine temperature and density profiles; TS consists of core and edge subsystems covering the upper half of the plasma with 54 spatial points, sharing the laser beams of four Nd:YAG lasers with $30 \mathrm{~Hz}$ repetition 
rate each [37],

- six pinhole cameras with 20 channel AXUV bolometers applied for measurements of spatial distribution of total plasma radiation with high $(1 \mu \mathrm{s})$ temporal resolution [38]; the AXUV bolometers often proved helpful in understanding the RE evolution, however, their failures during disruptions present a common problem,

- three high-resolution minispectrometers (combined range $250-1080 \mathrm{~nm}$ ) also proved valuable in the preliminary interpretation of the RE processes related namely to injected gas ionisation and excitation - the spectroscopic data have significant potential for further intensive data analyses, provided that a sophisticated transport model with atomic data is available.

\section{Results of the RE research at COMPASS}

The early RE dedicated experiments at COMPASS tokamak surprisingly proved permanence of the RE population in the HXR data even at relatively high plasma densities $n_{\mathrm{e}} \sim 4-6 \cdot 10^{19} \mathrm{~m}^{-3}$ in the D-shaped plasmas [15]. This might be partly sustained by the secondary avalanching process, for the key role of the seed RE population from the ramp-up phase at these densities was demonstrated. Indeed, a simple secondary puff in the fuelling within the current ramp-up can inhibit the early $\mathrm{RE}$ population. In that case, RE appear in the current plateau only at very low plasma densities, approximately $n_{\mathrm{e}}<2 \cdot 10^{19} \mathrm{~m}^{-3}$ [33]. This density value corresponds to the critical field $E / E_{\mathrm{c}} \geq 10$ where $E_{\mathrm{c}}$ is defined by Eq. (1). The favourably high experimental value of the critical field as compared to the predicted one is in agreement with other facilities [3, 27, 29, 30] and it is probably linked to the magnetic field fluctuations, although other interpretations can be found, see e.g. [39, 40].

In the extremely low density RE dominated discharges, record length of COMPASS discharges were achieved (above $1 \mathrm{~s}$ ) and, importantly, synchrotron radiation of the RE beam was observed [41]. From these measurements in combination with modelling, the upper limit of RE energy at COMPASS was determined to be $40 \mathrm{MeV}$ [41. In this respect, HXR photons of up to $7 \mathrm{MeV}$ were recorded during the RE experiments, approaching the limit given by the size of the scintillation detector. Herefrom it can be concluded that the maximum RE energies can reach a few tens of $\mathrm{MeV}$.

Post-distruption RE beams were observed at COMPASS following Massive Gas Injection (MGI) into the current ramp-up phase [33]. This scenario is particularly important due to its relevance to ITER, however, it suffers with low reproducibility which is probably linked to the role of a precise field helicity at disruption. Clear filamentation was documented in the RE beam following the MGI triggered disruption in the fast visible camera data [42. The filaments connected with the collapse of magnetic surfaces were also captured by the V-ECE, HXR and by magnetic diagnostics [42, 43]. The same data show rather stable vertical position of the RE beam and its initially slow instability in the radial position that can be improved with a dedicated controller as mentioned below. Besides, if elongated, the RE beam usually terminates in vertical 


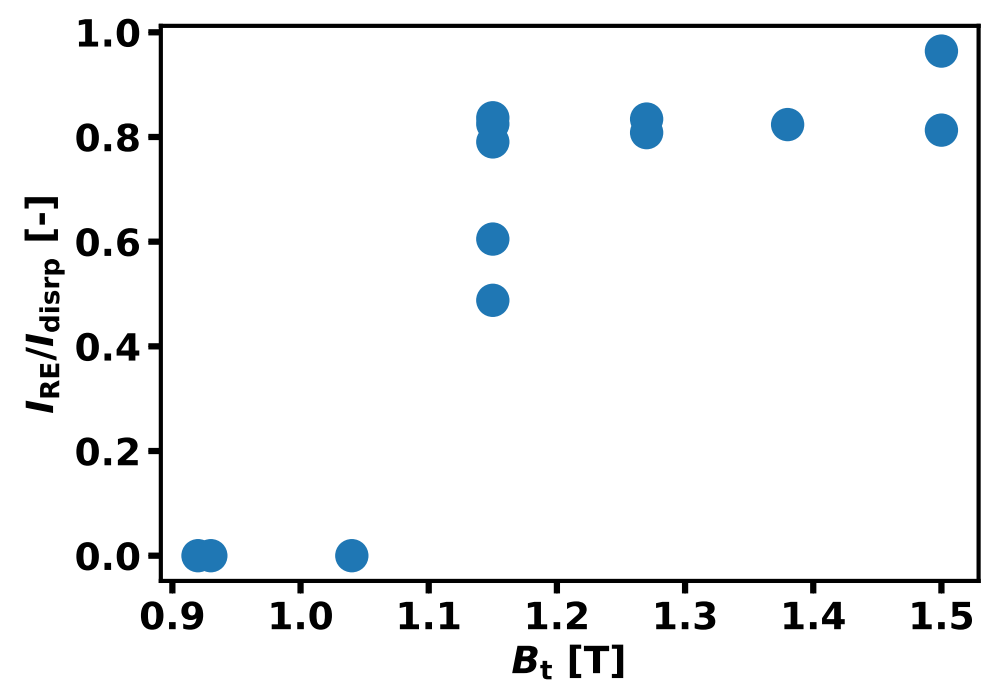

Figure 2. The RE current amplitude following an MGI disruption, normalised to pre-disruption plasma current, as a function of the toroidal magnetic field. A clear threshold level at about $B_{T}=1.15 \mathrm{~T}$ is disclosed.

instability. In order to clarify the role of magnetic field in this scenario, a scan of the RE beam current as a function of the $B_{T}$ field amplitude was measured recently, see Fig. 2. In this scan, the direction of the $I_{p}$ was anti-clockwise so the $B_{t}$ and $I_{p}$ were antiparallel. As expected according to [44] a clear threshold level of the $B_{T}$ field for generation of the RE beam at COMPASS was observed, although at a rather low levelat about $B_{T}=1.15 \mathrm{~T}$. The reproducibility was also improved by optimising the radial position reference and in tests of the new RE controller [19].

In another scenario, inspired by the TCV RE experiments [27, 29, 30] the plateau plasma current was converted to a strong RE population following an impurity gas puff. The injection was applied on the high field side in the divertor region, with impurity density comparable to the plasma density, $n_{\mathrm{e}} \approx 1 \cdot 10^{19} \mathrm{~m}^{-3}$. The conversion proved to be highly reproducible and robust, providing a solid base for a systematic experimental work. Results presented in the following subsections, including the Resonant Magnetic Perturbation (RMP) experiments, were achieved in this scenario.

\subsection{Multiple effects of gas injection}

An impurity gas puff ( $\mathrm{Ar}$ or $\mathrm{Ne}$ ) into the current flat-top of very low density discharges $\left(n_{\mathrm{e}} \approx 10^{19} \mathrm{~m}^{-3}\right)$ causes a rapid thermal quench, clearly visible in the Thomson scattering data. Depending on the pre-puff density, RE seed amount and gas injection amount, the thermal quench is sometimes intertwined with a current quench that terminates the discharge promptly without any RE beam generation or, more often, the current level shows no abrupt change while the HXR radiation steeply rises. This implies that a major part of the current is converted into the RE population. Consequently, the final 
part of this scenario i.e. the discharge following the plasma thermal quench due to the impurity puff will be referred to as the RE dominated discharge.

A surprising observation was done with respect to the MGI timing in experiments with a fixed amount of injected gas particles at approx. the threshold amount $10^{21} \mathrm{~m}^{-3}$, given $B_{T}=1.5 \mathrm{~T}$ and constant fuelling. In these experiments, the terminal current quench appeared only until a threshold instant in the current plateau. A later timing of the MGI single inlet resulted in a RE dominated discharge with a substantially longer current decay time. This robust result was observed in discharges \#16670 - \#16677 with a threshold time $1090 \mathrm{~ms}$ (i.e. $130 \mathrm{~ms}$ after the plasma breakdown) and precision $<2 \mathrm{~ms}$. This can be interpreted in terms of a significant role of parameters of the pre-disruption RE population. Notice that both RE density and energy increase steadily during the current plateau due to low plasma density and the avalanching effect. However, the avalanche multiplication of the RE population shall be less significant for the COMPASS conditions compared to larger facilities. The runaway fraction originating from knockon collisions is $<5 \%$ for $E / E_{\mathrm{c}} \approx 90$ and $T_{\mathrm{e}} \approx 0.6 \mathrm{keV}$ and the avalanche time ( $\tau_{\text {ava }}$ ) can reach up to hundreds of milliseconds, as shown in [45]. In the case of smaller $E / E_{\mathrm{c}}$ (10-50), the RE avalanche fraction can significantly increase and $\tau_{\text {ava }}$ decrease, see [46].

In order to quantify the role of the impurity gas in the RE dominated discharges, a novel current control technique was introduced: Following the gas puff, the power control of the tokamak was switched from the constant plasma current feedback to the constant Central Solenoid current regime. As a result, the induced toroidal electric field was actively kept close to zero $\left(U_{\text {loop }} \approx 0 \mathrm{~V}\right)$ in this scenario. This is significant for validation of theory and models as it allows to study an acceleration-free decay of the RE population. The subsequent results - including the RMP experiments in Sec. $2.2-$ were collected in this scenario, if not stated otherwise.

In the slow, RE dominated current decay following the thermal quench the current decay rate (CDR) was observed to be a function of the injected gas amount, see Fig. 3. It is apparent that with a low impurity density, the RE dominated discharge can sustain CDR less than $1 \mathrm{MA} / \mathrm{s}$ in neon. The results for argon, with lower ionisation energy than neon, indicate approx. double CDR as compared to neon due to the stronger drag force. In order to further elaborate on these results, experiments with plasma deuterium fuelling in the RE dominated discharge were undertaken, see Fig. 4 . In the figure, three different scenarios after Ar gas puff are compared, documenting the following current decay mechanisms:

(i) The RE dominated discharge with constant current feedback control and D fuelling (in red line, mid-grey in printed version). Deuterium supports the current feedback by neutralising the argon impurity and therefore by decreasing the RE drag. This interpretation is strengthened by fast visible camera data that shows temporal decrease of the Ar II radiation. The discharge suffers with instabilities linked to rational magnetic field surfaces, accompanied by filamentation visible in the camera data as well as by peaks in other signals [42]. With a termination of the deuterium fuelling and due to the Ohmic heating, the Ar impurity takes over again as a strong 


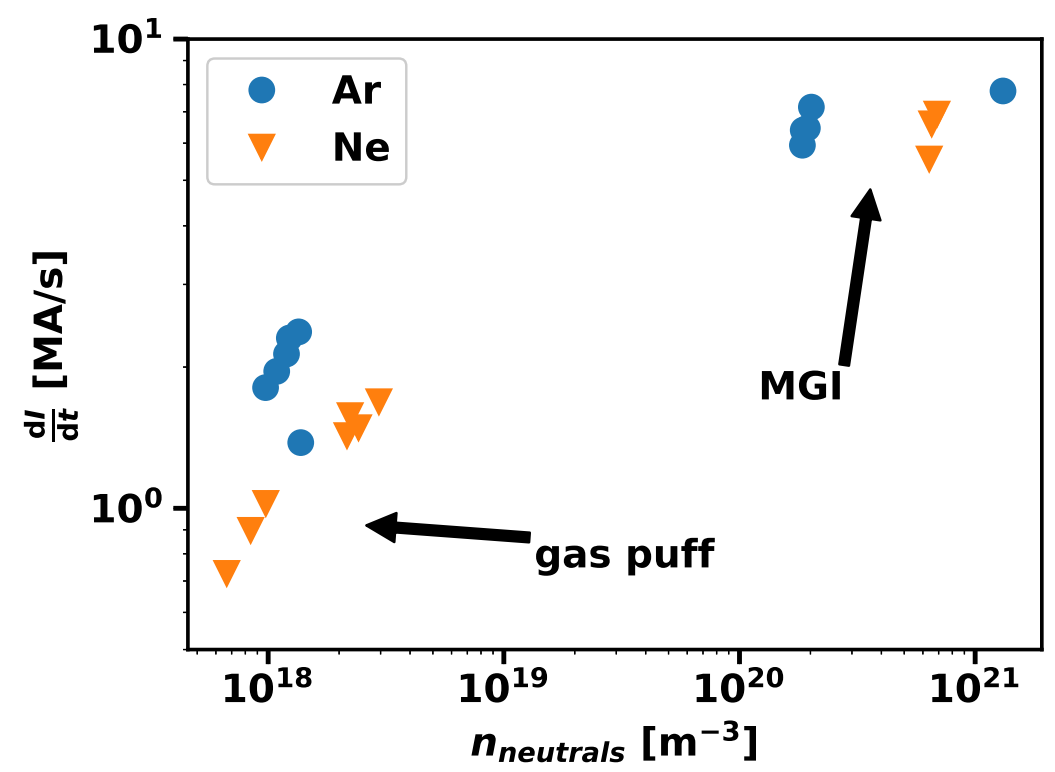

Figure 3. Current decay rate in the gas amount scan, showing both gas puff and MGI into a late stage of the current plateau $\left(I_{p}=140 \mathrm{kA}\right)$. In this scan, current in the COMPASS Central Solenoid was fixed at a constant level in order to maintain $U_{\text {loop }}$ close to zero.

radiative component.

(ii) The RE dominated discharge with zero $U_{\text {loop }}$, with no additional fuelling (in blue line, black in printed version). The current decay in this scenario is smooth, with a relatively high CDR.

(iii) The RE dominated discharge with zero $U_{\text {loop }}$ and deuterium fuelling (in green line, light grey in printed version). Deuterium neutralises the argon impurity, which considerably decreases the electron density and RE drag and results in a substantially lower CDR. Notice that in this scenario the RE dominated discharge eventually lives longer than the similar discharge with the current feedback.

The above understanding is further supported by HXR data evolution, in particular in the case of the current feedback where the high levels of the HXR radiation (leading to saturation of the detector) reflect high energies of the lost RE due to high $U_{\text {loop }}$. Furthermore, evolution of the total radiated power (bottom frame in the figure) demonstrates that the radiative losses are higher in the case of the current feedback than in the other scenarios, and that the losses strongly increase when deuterium fuelling stops. The total losses were determined from $2 \mathrm{D}$ tomography of the AXUV signals calculated by the Minimum Fisher Regularisation (MFR) tomography [47]. A hollow belt of the radiation profile following the gas puff was clearly reconstructed by the tomographic analysis. The MFR tomography application in this task present a straightforward continuation of our initial contributions to the dedicated RE studies at 

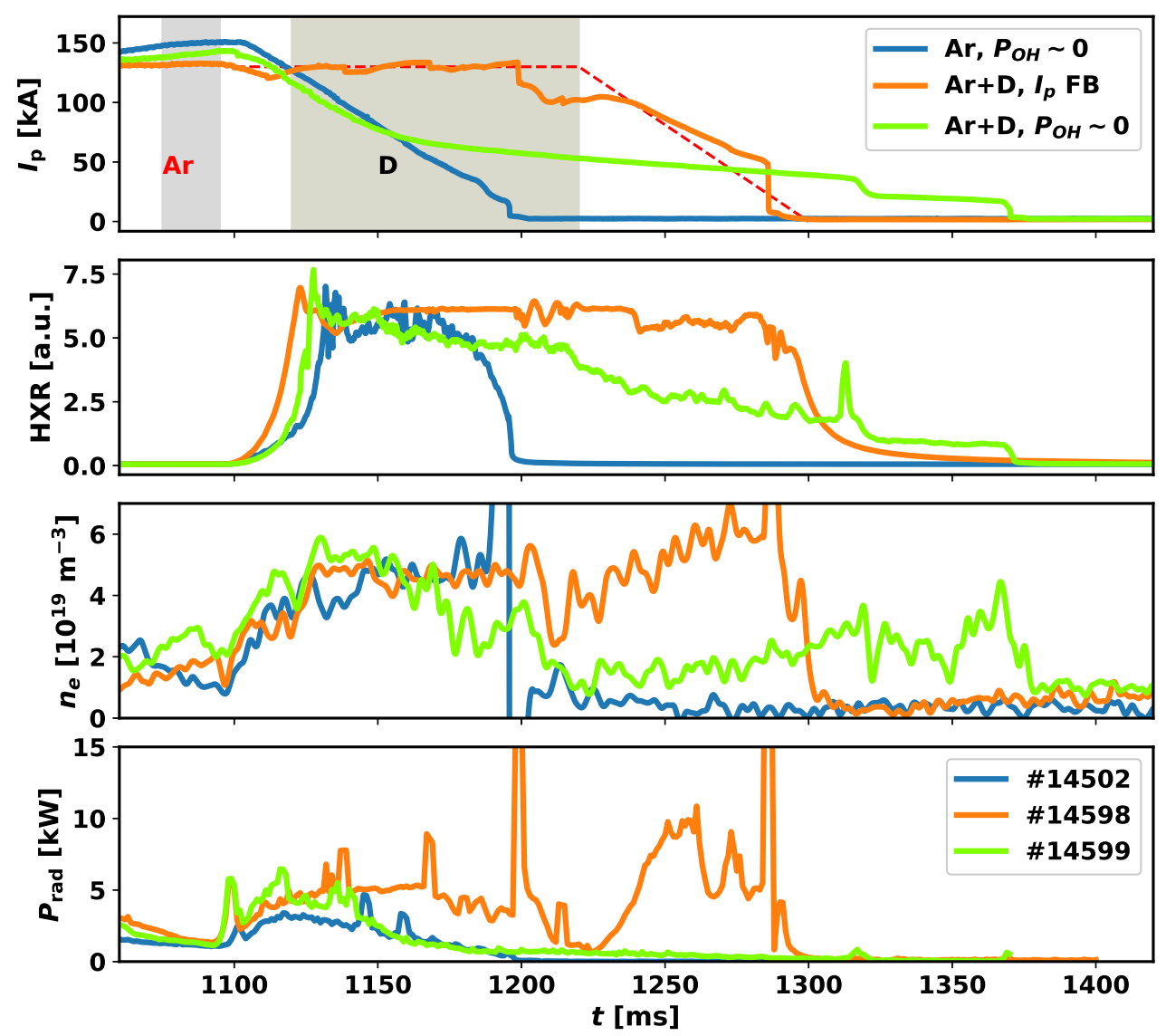

Figure 4. Three scenarios of RE dominated discharge following the thermal quench due to the Ar gas puff into the low density current plateau at COMPASS. Red (medium grey in print) line: current feedback and additional deuterium fuelling (current reference in dashed line), blue (black) line: zero $U_{\text {loop }}$ with no additional fuelling, green (light grey) line: zero $U_{\text {loop }}$ with current feedback and additional fuelling. Note that with the thermal quench due to the gas puff, $T_{e}$ drops below $10 \mathrm{eV}$.

JET, see [48].

Typical CDRs of discharges with large to dominant RE population are compared in Tab. 1 .

Numerical simulations of selected quiescent RE flat-top discharges with zero $U_{\text {loop }}$ scenario and high $Z$ impurity seeding were performed with the linearized Fokker-Planck solver CODE [49, 50]. The simulations included the effect of partial screening [39, 51], large-angle collisions [6] and self-consistent calculation of the electric field, but no radial transport losses due to magnetic field fluctuations. Including partial screening gives more accurate results than previous theoretical predictions (the ratio $E / E_{\mathrm{c}}$ is more than 2 times closer to the $E / E_{\mathrm{c}}$ value from experiment if partial screening is included than without), however, there is still a factor of 3-5 discrepancy between the simulation and experiment. The discrepancy might be explained by RE transport losses due to 


\begin{tabular}{|l|l|l|}
\hline Discharge termination & CDR [MA/s] & Effects \\
\hline Natural decay & $0.1-0.2$ & moderate HXR \\
\hline Ar/Ne gas puff + D & $0.2-0.3$ & $\begin{array}{l}\text { intensive HXR, changes } \\
\text { in colour of visible light }\end{array}$ \\
\hline Ar/Ne gas puff & $1.5-2.5$ & faster decay, saturated HXR \\
\hline Ar/Ne gas puff \& RMP & $5.0-9.0$ & fast decay, saturated HXR \\
\hline Ar/Ne MGI & $6.0-9.0$ & $\begin{array}{l}\text { intensive line radiation, steep } \\
\text { but shorter burst of HXR }\end{array}$ \\
\hline Plasma disruption & $200-700$ & a very short HXR burst \\
\hline $\begin{array}{l}\text { Disruption of RE dominated plasma } \\
\text { without impurity gas }\end{array}$ & $4000-7000$ & $\begin{array}{l}\text { immediate, localised } \\
\text { damage, high peak in HXR }\end{array}$ \\
\hline
\end{tabular}

Table 1. Typical current decay rates at COMPASS in different discharge terminations.

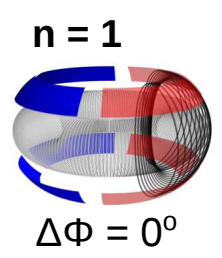

EW orient.

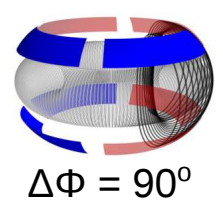

NS orient.

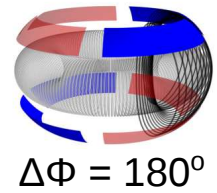

WE orient.

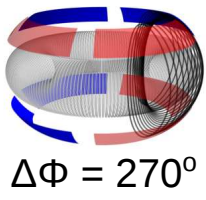

SN orient.

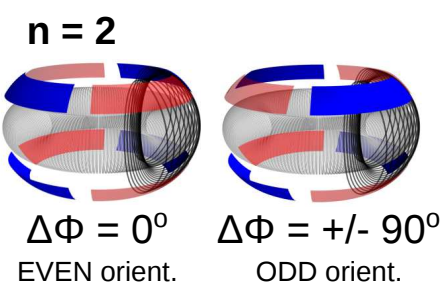

EVEN orient. ODD orient.

Figure 5. Configuration of the RMP coils in the RE experiments at COMPASS. The red (light grey in print) and blue colors (dark grey) correspond to positive (direction out of plasma) and negative $B_{r}$ field perturbation, respectively.

magnetic fluctuations. Note that in all the above studied cases fluctuations in the radial magnetic field $\left(\delta B / B \geq 5.10^{-4}\right)$ were observed.

\subsection{RE in perturbed magnetic fields}

The key role of magnetic field perturbations on the RE losses was clearly identified already in past RE research at COMPASS and reported in [33]. Among others, an undisputable link between plasma MHD activity and the RE confinement was documented, and a relation between the switching of the power supply and modulation of the HXR radiation was revealed. Next, a minor modulation in plasma position due to the feedback system properties proved to produce a well correlated effect between HXR radiation and infrared radiation from the limiter, which directly confirmed that RE interaction with the limiter makes a significant contribution to the overall HXR radiation intensity [33].

In this subsection, the main results from recent RE experiments with RMP system at COMPASS [52, 53] are presented. The work was partly inspired by recent work of the ASDEX Upgrade team in RMP experiments on post-disruption RE beam [54. Subsequently the RMP configurations were limited to the low field side offmidplane coils only, see Fig. 5, while the effect of coils at high field side midplane, 


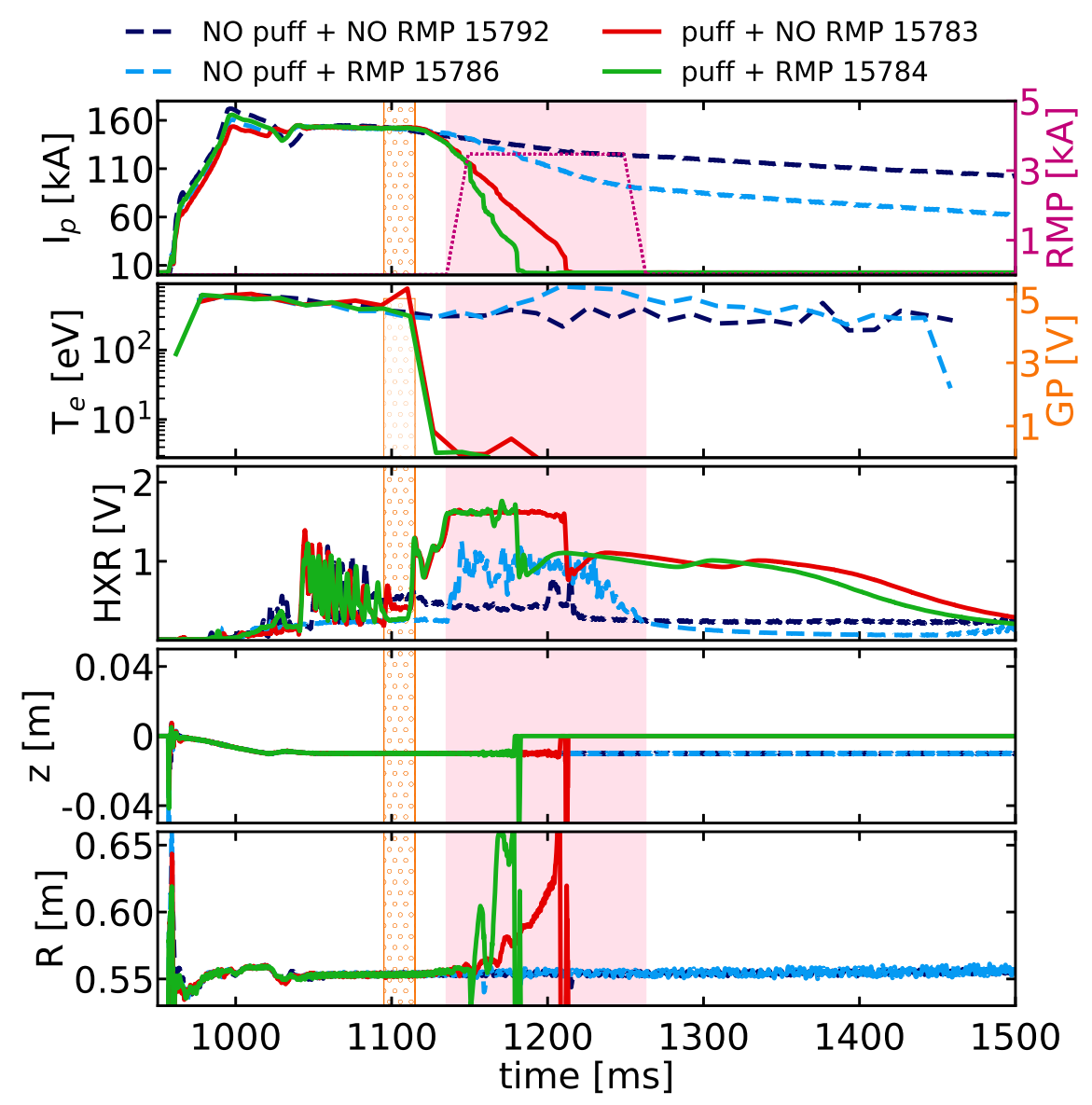

Figure 6. Termination of the $\mathrm{RE}$ dominated discharge with zero $U_{\text {loop }}$, showing consequences of argon puff at $5 \cdot 10^{18}$ particles, and/or the $n=1$ RMP field.

low field side midplane, or top and bottom may be subject to the future RE research. The perturbation can reach up to $B_{r} / B_{T} \approx 10^{-2}$. However, the magnitude of the perturbation was always kept below the empirical threshold of MHD mode locking in order to avoid significant perturbation of background plasma. Similarly to [54], we study the effect of the RMP on RE beam in context of coupling of the RMP to rational surfaces $m / n$ of the magnetic equilibrium. However, presence of external magnetic perturbation leads to induction of screening currents on these surfaces [55] that alter this coupling. Thus, the screened RMP has been calculated using a resistive MHD code MARS-F [56].

In order to achieve high reproducibility under well-defined conditions, the scenario described in Subsec. 2.2 was exploited. The different effects of gas puff and RMP field in the RE dominated discharge following a gas puff and featuring an active control of the zero $U_{\text {loop }}$ is summarised in Fig. 6. Although the thermal quench due to the gas puff obviously played a dominant role in the CDR (i.e. in the discharge length), it is also clear that the RMP effect systematically increased the RE losses - as observed in the HXR data - and therefore caused a faster CDR. The effect of the RMP field on the plasma 

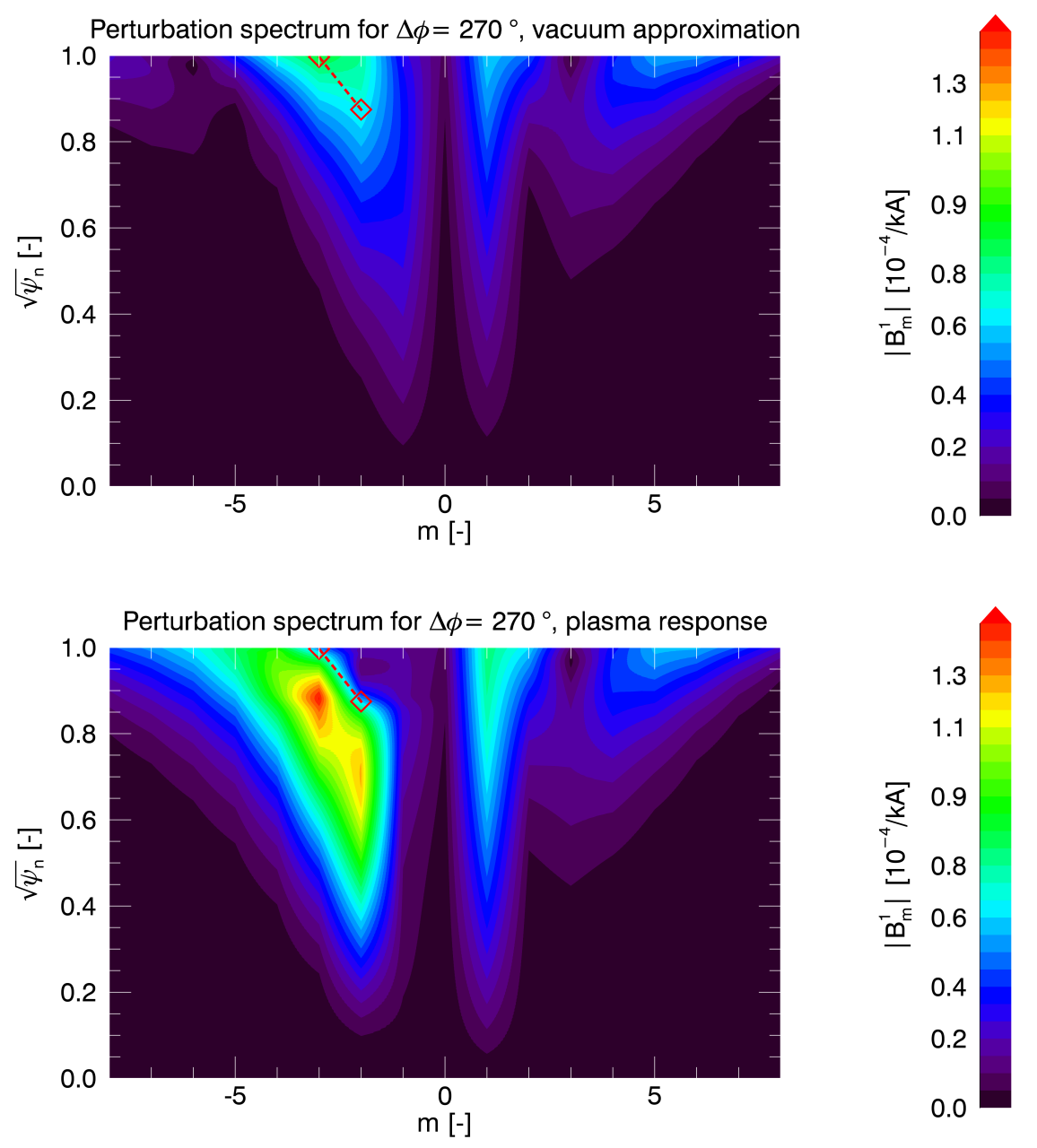

Figure 7. Fourier decomposition of dimensionless perturbation field magnitude $\left|\mathbf{B}^{1}\right|=\left|\frac{\mathbf{B}_{\text {pert }} \cdot \nabla \Psi}{\mathbf{B}_{\text {equi }} \cdot \nabla \Phi} \frac{q}{R^{2} B_{0}}\right|$ into poloidal modes as a function of radius $\left(\sqrt{\Psi_{n}} \approx r / a\right)$. Quantity normalized to $I_{\text {coil }}$ of perturbation coils at $\Delta \Phi=270^{\circ}$. Positions of equilibrium rational surfaces are shown in diamond symbols. Top plot - vacuum approximation, bottom plot - plasma screening included.

center position in vertical and radial directions is visible in two bottom panels. The vertical position remains stable, whereas the radial position is unstable as mentioned previously. In the figure, CDR with phase $\Delta \Phi=270^{\circ}$ of the top with respect to the bottom RMP coils is presented (see Fig. 5). The role of the phase was determined in a separate scan of the four possible $\Delta \Phi$ angles. It has been observed in the experiment that the most pronounced effect of RMP on CDR, similarly to [54], is associated with the strongest coupling of screened RMP to the equilibrium rational surfaces. Furthermore, comparison of vacuum perturbation spectrum to the one including plasma response in Fig. 7 reveals that under these conditions a strong kink mode amplification due to plasma screening appears, as it is indicated by amplification of components at $|\mathrm{m}|$ higher than those of rational surfaces (shown by red symbols). This is also in agreement with 


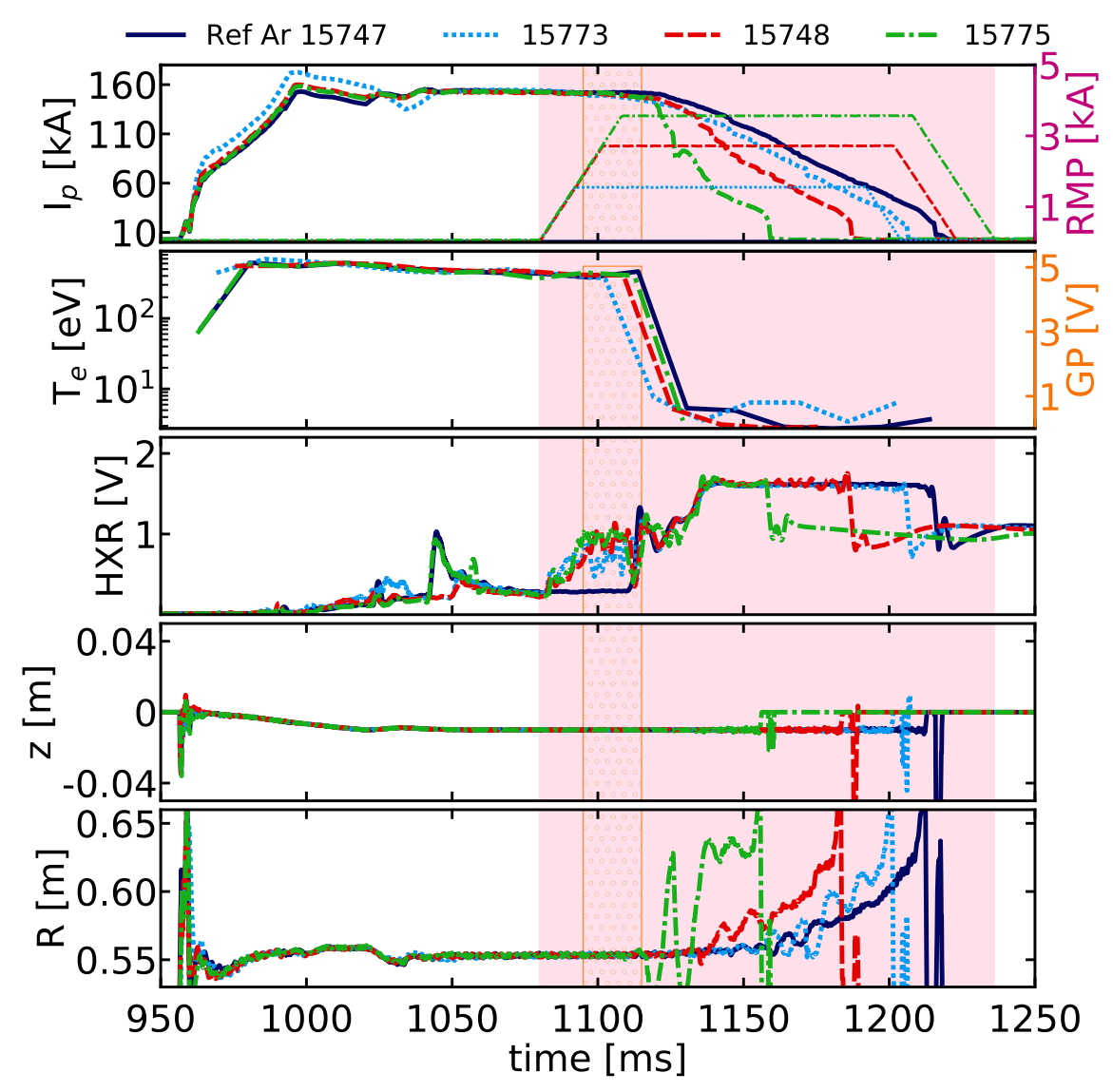

Figure 8. Observed increase of CDR with increase of the RMP amplitude. The scan was performed for the argon puff at $5 \cdot 10^{18}$ particles, the partly resonant phase $\Delta \Phi=270^{\circ}$ and zero $U_{\text {loop }}$.

observations in [54].

Next, experiments scanning the amplitude of the RMP were performed. The highest RMP amplitude in the most resonant (from screened RMP-equilibrium coupling point of view) $\Delta \Phi=0^{\circ}$ phase resulted in a fast disruption with sudden current quench and no RE dominated discharge for RMP amplitude $>1.1 \mathrm{kA}$ in case of argon and $1.7 \mathrm{kA}$ in case of neon, with no RE dominant phase. Therefore, the second most resonant phase $\Delta \Phi=270^{\circ}$ and the argon puff at $5 \cdot 10^{18}$ particles was used in the published amplitude scan, see Fig. 8. Additionally, in this set of experiments the RMP coils were switched on well before the gas puff to investigate influence of the perturbed field on the current plateau. The figure shows that with increasing amplitude of current in the RMP coils the CDR increased as expected, and that increased RE losses due to the RMP field occurred even before the thermal quench, as can be seen from the HXR signal. These data demonstrate presence of well-confined RE in the current plateau phase, i.e. before the thermal quench, which acts as the RE seed in the RE dominated discharge. The 


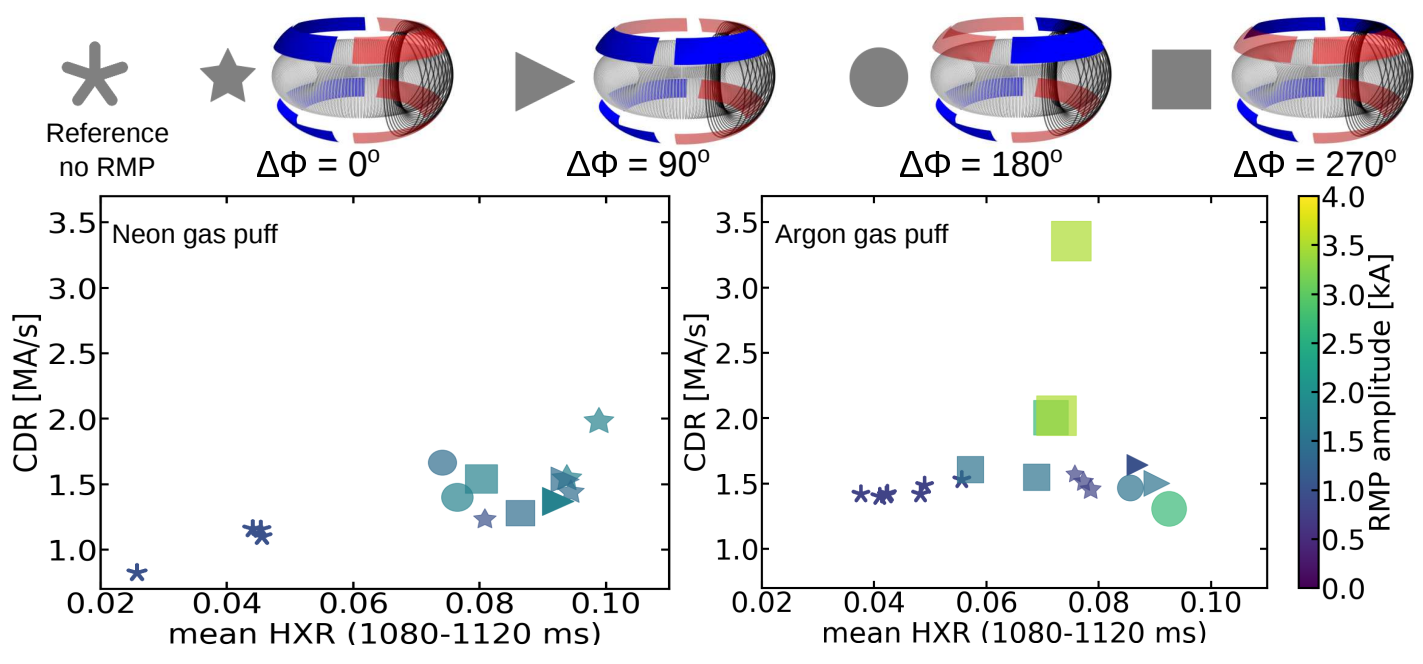

Figure 9. Overview of results obtained with application of $n=1$ RMP field from before disruption to the end of the RE dominated current decay at COMPASS. Current decay rate for the four different RMP phases is shown as a function of the early HXR radiation level.

seed can be partly lost due to the RMP field.

The overview statistics of the RE experiments in all four phases of the $n=1 \mathrm{RMP}$ fields and either argon or neon induced thermal quench at COMPASS is presented in Fig. 9. The figure shows CDR as a function of average early level in the HXR data (from $1080 \mathrm{~ms}$ to $1120 \mathrm{~ms}$, avoiding signal saturation). No RMP field was applied in the reference discharges. The strong correlation between the early HXR intensity and the CDR is apparent in particular in the neon case: the more intense the HXR radiation, the faster the CDR. It is also apparent that the fastest decay was achieved for $\Delta \Phi=0^{\circ}$ for neon and $\Delta \Phi=270^{\circ}$ for argon. In the case of argon, the CDR seem to be almost independent of the early HXR level except for the highest RMP current amplitudes in the $\Delta \Phi=270^{\circ}$ phase, where the CDR was substantially faster.

Systematic experimental studies were also performed for $n=2$ RMP with two different phases: the even phase with $\Delta \Phi=0^{\circ}$, and the odd phase with $\Delta \Phi= \pm 90^{\circ}$. Higher losses of RE (linked to more intense HXR radiation and higher CDR) were observed in the odd phase, which was correlated with onset of a rotating MHD mode (detected by an in-vessel local poloidal magnetic field probe) and the fluctuations of HXR signal observed upon powering the RMP coils. However, a still stronger effect is expected at $\Delta \Phi=45^{\circ}$. As in the $n=1$ RMP amplitudes $>3.25 \mathrm{kA}$ led to immediate current quench with no surviving $\mathrm{RE}$ in the case of the argon gas puff, while in the case of the neon gas puff the RE beam survived even the highest RMP amplitude.

It should be pointed out that due to the specific proximity of RMP coils to plasma at COMPASS, and their relatively high coil currents, it is possible to exert a strong perturbation (not necessarily resonant) to plasma equilibrium, in comparison to other devices. As it is shown in Fig. 8, this is responsible for the increase in the CDR. 
Although RMP is not foreseen as a RE mitigation scheme due to minor effect on RE at large facilities, the above presented results are relevant for validation of models which shall improve understanding of interaction of RE with perturbed magnetic field.

\subsection{Complementary research programme}

The complementary research programme in support of RE experiments is fostered at COMPASS in order to build up the basis for further progress. The programme consists of development of modelling tools, development and integration of dedicated novel diagnostics and implementation of new control methods into the discharge scenario.

The present modelling of the RE dynamics at COMPASS [46] relies on fast transport code METIS [57], which has been recently coupled to the $1 \mathrm{D} 2 \mathrm{~V}$ relativistic bounceaveraged kinetic Fokker-Planck solver LUKE [45, 58]. As a result, radial electric field and estimate of the fraction of current carried by RE can be calculated selfconsistently as well as dynamics and distribution function of the RE. Modeling of effects of impurity seeding, transport and radiation losses on the RE current decay and future benchmarking with experiments are under consideration within a broad international collaboration. As another example, contributions to coupling of dynamics of energetic particles and MHD modes modeling in tokamaks have been developed. The coupling is based on simulations of transport of runaway electrons in the presence of MHD perturbation with the Hybrid Gyrokinetic Code [59]. Fundamental theory works with respect to $\mathrm{RE}$ distribution function and radiation have been also fostered in the team since 2018. In the longer term, the new COMPASS Upgrade project presents an important challenge to the team due to preparation of the prospective programme, with an exciting potential [60].

The RE related work also contributes to development and exploitation of new dedicated diagnostics. Three channel Cherenkov detector, under development in the Polish NCBJ institute [61, 62] makes a regular part of the RE campaigns with a particular advantage of sensitivity to lower energy RE seed. A new method of observation of the confined RE electrons at 50-140 keV using vertical ECE measurements on the 3rd harmonics proved to provide new valuable insights into the RE dynamics and its interaction with the core plasma [43]. Next, a 2D soft X-ray matrix detector MediPix is under detailed tests at COMPASS as it would provide invaluable information on spatial distribution of the RE interaction with the first wall [63]. The same detector shall be used in the planned solid pellet injector experiments. Last but not least, exploitation of the RE Infrared Spectroscopy detector REIS on loan is foreseen [64.

In the field of implementation of the new control methods, the aim is to develop a reliable active control of the position and current in RE dominated discharge and/or for pure RE beam in tokamaks, with the motivation to minimise the RE interaction with the first wall. At COMPASS, with support of to expertise of foreign collaborators, the MARTe real-time framework was installed and current quench detector was implemented as well as the radial position and plasma current reference policies [19]. With the present 
encouraging results, the development of the RE control is bound to continue.

\section{Conclusions}

Research into foreseeable methods of the RE mitigation presents a high priority task in support of ITER. The COMPASS tokamak has contributed extensively to this research recently, in particular in the field of experiments focused on RE interaction with injected gas and with magnetic perturbation. The shared expertise with other facilities, including the possibility to participate in the JET, ASDEX Upgrade and TCV RE studies were instrumental.

Role of filamentation due to the field line helicity and role of toroidal magnetic field intensity were studied in the MGI triggered post-disruption RE beam experiments in the current ramp-up phase. Majority of the RE research results presented in this contribution was achieved with gas puff into low density current plateau phase of the discharge, which reliably resulted in a rapid thermal quench of the plasma followed by a slow current decay of the RE dominated discharge. Better understanding of the RE mitigation in these experiments was achieved due to introduction of the zero $U_{\text {loop }}$ via an active control of the constant current in the Central Solenoid of COMPASS. Differences between application of argon and neon were identified in evolution of the RE dominated discharges and attributed to their different ionisation potential. Studies of the role of the ionisation potential with respect to the discharge conditions were complemented by deuterium fuelling of the RE dominated discharge. It was concluded that the additional fuelling results in cooling of the background plasma, which effectively improves the RE confinement.

A large number of experiments with RMP was performed in the same scenario. In all the experiments the RMP field led to increased losses of RE. The undertaken scans in the RMP phase agreed well with predictions of the MARS-F interpretative MHD model, showing that most important RE losses (and, subsequently, the fastest current decays in the RE dominated discharges) were associated with the coupling between the plasma-screened RMP and the magnetic equilibrium rational surfaces. The RMP coils were powered both before and after the impurity gas puff, demonstrating that the RE deconfinement due to perturbation appears in the RE dominated disruption independently of the same effect on the hot plasma. In the case of large RMP amplitudes, the perturbation followed by gas puff often led to immediate current quench without any surviving RE beam. With this exception, the scan on RMP amplitudes demonstrated that the higher the RMP amplitude, the higher the CDR in the RE dominated discharges.

Interpretation of the $\mathrm{RE}$ experiments is challenging partly due to inadequate features of common tokamak diagnostic systems for the RE research as well as due to multiple complexities in theory and modelling of fast particles in magnetically confined

plasmas. Therefrom, research and development of RE relevant diagnostics and computer models make an integral part of the RE studies at COMPASS. Scenario development 
based on novel position and current controllers presents another opportunity for the COMPASS RE research complementing the experimental studies. Indeed, the RE control seems feasible with the tokamak field actuators, while it may prove to have an important potential for the required RE mitigation schemes.

\section{References}

[1] Connor J.W., Hastie R.J., 1975 Nucl. Fusion 15415

[2] Dreicer H., 1959 Phys. Rev. 115238

[3] Granetz R. S. et al. 2014 Phys. Plasmas 21072506

[4] Gurevich A.V., Milikh G.M., Roussel-Dupre R. 1992 Phys. Lett. A 165463

[5] Rosenbluth M.N. and Putvinski S.V., 1997 Nucl. Fusion 371355

[6] Embreus O. et al., 2018 J. Plasma Phys. 84905840102

[7] Knoepfel H., Spong D.A. 1979 Nucl. Fusion 19785

[8] Popovich Z. et al., 2016 Phys. Plasmas 23122501

[9] Paz-Soldan C. et al., 2014 Phys. Plasmas 21022514

[10] Smith H.M., Verwichte E, 2008 Phys. Plasmas 15072502

[11] Papp G. et al., 2013 Nucl. Fusion 5312301

[12] Hollmann E.M. et al., 2015 Phys. Plasmas 22021802

[13] Boozer A. H., 2017 Nucl. Fusion 57056018

[14] Feher T. et al., 2011 Plasma Phys. Control. Fusion 53035014

[15] Mlynar J. et al., 2015 Proc. 42nd EPS Conf. Plasma Phys. (Lisbon) ECA Vol. 39E P4.102

[16] Lehnen M. et al., 2015 J. Nucl. Mater. 46339

[17] Riccardo V et al., 2010 Plasma Phys. Control. Fusion 52124018

[18] Hender T.C. 2007 Nucl. Fusion 47 (6) S128-S202

[19] Carnevale D. et al., 2018 Plasma Phys. Control. Fusion this special issue

[20] Paz-Soldan C. et al., 2017 Phys. Rev. Lett. 118255002

[21] Tinguely A.R. et al., 2018 Nucl. Fusion 58 (7) 076019

[22] Hoppe M. et al., 2018 Nucl. Fusion 58 (8) 082001

[23] Hoppe M. et al., 2018 Nucl. Fusion 58026032

[24] Boozer A. H., 2015 Phys. Plasmas 22032504

[25] Stahl A. et al., 2016 Nucl. Fusion 56112009

[26] Reux C. et al. 2015 Nucl. Fusion 55129501

[27] Papp G. et al. 2016 Proc. 26th IAEA FEC (Kyoto, Japan) IAEA CN 234 EX/9-4

[28] Pautasso G. et al., 2017 Plasma Phys. Control. Fusion 59014046

[29] Decker J. et al., 2018 Plasma Phys. Control. Fusion submitted

[30] Coda S. et al. Nucl. Fusion 57102011

[31] Esposito B. et al. 2016 Proc. 26th IAEA FEC (Kyoto, Japan) IAEA CN 234 EX/P8-27

[32] Vlainic M. et al. 2015 J. Plasma Phys. 81475810506

[33] Ficker O. et al. 2017 Nucl. Fusion 57 076002P

[34] Plyusnin V.V. et al. 2018 Nucl. Fusion 58016014

[35] Panek R. et al. 2016 Plasma Phys. Control. Fusion 58014015

[36] Weinzettl V. et al. 2011 Fusion Eng. Des. 861227

[37] Bilkova P. et al., 2018 J. Instrum. 13 C01024

[38] Mlynar J. et al., 2012 Rev. Sci. Instrum. 83 10E531

[39] Hesslow L. et al., 2017 Phys. Rev. Lett. 118255001

[40] Stahl A. et al., 2015 Phys. Rev. Lett. 114115002

[41] Vlainic M. et al., 2015 Proc. 42nd EPS Conf. Plasma Phys. (Lisbon) ECA Vol. 39E P4.108

[42] Ficker O. et al., 2017 Proc. 44th EPS Conf. Plasma Phys. (Belfast) ECA Vol. 41F P5.126

[43] Farnik M. et al., 2018 Proc. 45th EPS Conf. Plasma Phys. (Prague) ECA Vol. 42A P1.1010 
[44] Zeng L. et al., 2013 Phys. Rev. Lett. 110235003

[45] Nilsson E. et al., 2015 Phys. Plasmas 23711

[46] Macusova E. et al., 2018 Proc. 45th EPS Conf. Plasma Phys. (Prague) ECA Vol. 42A P1.1019

[47] Odstrcil M. et al., 2012 Nucl. Instrum. Methods Phys. Res. A 686156

[48] Plyusnin V.V. et al., 2006 Nucl. Fusion 46 (2) 277

[49] Landreman M. et al., 2014 Computer Physics Communications 185847 - 855

[50] Stahl A. et al., 2016 Nucl. Fusion 56112009

[51] Hesslow L. et al., 2018 Plasma Phys. Control. Fusion 60074010

[52] Cahyna P. et al., 2009 Nucl. Fusion 49055024

[53] Markovic T. et al., 2016 Nucl. Fusion 56092010

[54] Gobbin M. et al., 2018 Plasma Phys. Control. Fusion 60014036

[55] Denner P. et al., 2014 Nucl. Fusion 54064003

[56] Liu Y.Q. and Bondeson A., 2000 Phys. Plasmas 73681

[57] Artaud J.F. et al., 2010 Nucl. Fusion 50043001

[58] Decker J. et al., 2016 Plasma Phys. Control. Fusion 57095006

[59] Briguglio S. et al., 1995 Plasma Phys. Control. Fusion 58025016

[60] Panek R. et al., 2017 Fusion Eng. Des. 12311

[61] Rabinski M. et al. 2017 JINST 12 C10014

[62] Cerovsky J. et al., 2018 Proc. 45th EPS Conf. Plasma Phys. (Prague) ECA Vol. 42A P2.1006

[63] Neue G.et al., 2015 JINST 10 C04013

[64] Esposito B. et al., 2017 Plasma Phys. Control. Fusion 59014044

\section{Acknowledgments}

The work has been supported by the grant GA18-02482S of the Czech Science Foundation (GACR), the MEYS projects LM2015045, CZ.02.1.01/0.0/0.0/16_019/0000768 and carried out within the framework of the EUROfusion Consortium. It has also received funding from the Euratom research and training programme 2014-2018 under grant agreement No 633053 with the Co-fund by MEYS project number 8D15001. The views and opinions expressed herein do not necessarily reflect those of the European Commission. 